\title{
Lymphadenectomy is associated with poor survival in patients with gastrointestinal stromal tumors
}

\author{
Cong $\mathrm{Li}^{1 \#}$, Dongfang Su${ }^{2 \#}$, Chuanbo $\mathrm{Xie}^{3 \#}$, Qichen $\mathrm{Chen}^{4}$, Jianguo Zhou ${ }^{4}$, Xiaojun $\mathrm{Wu}^{1}$ \\ ${ }^{1}$ Department of Colorectal Surgery, ${ }^{2}$ Department of Clinical Nutrition, ${ }^{3}$ Department of Cancer Prevention Research, Sun Yat-sen University Cancer \\ Center, State Key Laboratory of Oncology in South China, Collaborative Innovation Center for Cancer Medicine, Guangzhou 510060, China; \\ ${ }^{4}$ Department of Hepatobiliary Surgery, National Cancer Center/National Clinical Research Center for Cancer/Cancer Hospital, Chinese Academy \\ of Medical Sciences and Peking Union Medical College, Beijing 100021, China \\ Contributions: (I) Conception and design: C Li, X Wu; (II) Administrative support: C Li, D Su; (III) Provision of study materials or patients: C Li, C \\ Xie; (IV) Collection and assembly of data: C Li, C Xie; (V) Data analysis and interpretation: C Li, C Xie; (VI) Manuscript writing: All authors; (VII) \\ Final approval of manuscript: All authors. \\ "These authors contributed equally to this work. \\ Correspondence to: Xiaojun Wu. Department of Colorectal Surgery, Sun Yat-sen University Cancer Center, State Key Laboratory of Oncology \\ in South China, Collaborative Innovation Center for Cancer Medicine, Guangzhou 510060, China. Email: wuxj@sysucc.org.cn; Jianguo Zhou. \\ Department of Hepatobiliary Surgery, National Cancer Center/National Clinical Research Center for Cancer/Cancer Hospital, Chinese Academy of \\ Medical Sciences and Peking Union Medical College, Beijing 100021, China. Email: zhoujg11@21cn.com.
}

Background: Current clinical practice suggests lymphadenectomy for gastrointestinal stromal tumor (GIST) patients with enlarged lymph nodes, but little is known about the influence of lymphadenectomy on long-term survival.

Methods: This population-based study consisted of 3,819 non-metastatic GIST patients diagnosed between January $1^{\text {st }}, 2001$, to December $31^{\text {st }}, 2015$, from the Surveillance, Epidemiology, and End Results (SEER) database. Kaplan-Meier methods and Cox proportion regression models were used to compare differences in overall survival (OS) and cancer-specific survival (CSS) between the lymphadenectomy group and nonlymphadenectomy group.

Results: Among the 3,819 GIST patients, 1,202 received lymphadenectomy and 2,617 did not receive lymphadenectomy. Lymphadenectomy was associated with poor OS (adjusted HR =1.25, 95\% CI: 1.06-1.47) and CSS (adjusted HR $=1.32$, 95\% CI: 1.07-1.64) in GIST patients. This was especially evident in GIST patients with a tumor size less than $2 \mathrm{~cm}(\mathrm{OS}, \mathrm{HR}=1.91,95 \% \mathrm{CI}: 0.79-4.60$ and CSS, HR $=6.37,95 \%$ CI: 1.85-21.90), who were more than 40 years old (OS, HR $=1.28,95 \%$ CI: $1.08-1.51$ and CSS, HR $=1.36,95 \%$ CI: $1.09-1.70)$, and with a stomach tumor (OS, HR $=1.39,95 \% \mathrm{CI}: 1.12-1.72$ and CSS, HR $=1.77,95 \%$ CI: $1.33-2.35)$.

Conclusions: In conclusion, contrary to what was previously presumed, lymphadenectomy was associated with an increased and not a decreased risk of mortality in GIST patients.

Keywords: Lymphadenectomy; gastrointestinal stromal tumors (GIST); overall survival (OS); cancer-specific survival (CSS); stratification analysis

Submitted Aug 22, 2019. Accepted for publication Sep 04, 2019.

doi: 10.21037/atm.2019.09.60

View this article at: http://dx.doi.org/10.21037/atm.2019.09.60 


\section{Introduction}

Gastrointestinal stromal tumors (GISTs) which originate from the cells of Cajal or the precursor of these cells, are the most common mesenchymal neoplasms of the gastrointestinal (GI) tract (1). They account for $1-2 \%$ of all GI tumors in the United States and can occur anywhere in the digestive tract. However, they frequently occur in the stomach and small intestine and less often in the esophagus, rectum, and colon (2). Approximately, $85 \%$ of GIST tumors express antigen CD117 which is a receptor tyrosine kinase coded by the KIT gene (3).

Surgery is the main treatment for regional and resectable GISTs (4). However, unlike other solid tumors, lymphadenectomy is not routinely performed during the surgical process as nodal involvement is rare in GIST patients (i.e., $1.1 \%$ to $3.4 \%$ of cases) (5). The current National Comprehensive Cancer Network (NCCN) guidelines advise lymphadenectomy to be performed only when the lymph nodes are suspected to be affected, as in the case of enlarged regional lymph nodes (6). Even so, enlarged regional lymph nodes might be free from cancer cells thereby making lymphadenectomy unnecessary. Most surgeons tend to perform lymphadenectomy during surgery, because it seems unlikely to increase the patient's risk of mortality. Therefore, it is a logical strategy to mitigate future intervention due to potential metastasis. However, lymphadenectomy is associated with increased surgical trauma and very likely to increase the risk of death (7).

Previous studies have shown that primary tumor size was positively associated with lymph node metastasis in solid tumors. For example, lymph nodes metastasis rates for T1a, $\mathrm{T} 1 \mathrm{~b}, \mathrm{~T} 1 \mathrm{c}$, and $\mathrm{T} 2$ stage breast cancer patients were $13.5 \%$, $20.4 \%, 35.9 \%$, and $50.0 \%$, respectively (8). Similar to breast cancer patients, we can hypothesize that GIST patients with a small primary tumor size (i.e., $<2 \mathrm{~cm}$ ) might have a low probability of metastasis, and thus do not need to undergo lymphadenectomy; however, patients with a large primary tumor (i.e., $>10 \mathrm{~cm}$ ) and a high probability of metastasis should be referred for lymphadenectomy. Compared to GIST patients of more than 40 years of age, younger GIST patients who have a relatively high risk of nodal involvement might benefit from lymphadenectomy (5). At present, these hypotheses have not been investigated.

In this study, we used the Surveillance, Epidemiology, and End Results (SEER) to examine whether lymphadenectomy was associated with poor survival rates in GIST patients and whether primary tumor size, patients' age, and tumor sites could be used as indicators for lymphadenectomy. Our study has implications in guiding surgeons to choose the most appropriate surgical treatments for GIST patients, specifically in relation to over-treatment.

\section{Methods}

\section{Study population}

We used data collected from the SEER project, which is a cancer registry system consisting of 18 regional registries covering $28 \%$ of the US population (9). SEER collects and codes data items using the North American Association of Central Cancer Registries (NAACCR). The code book can be downloaded from SEER's website (https://seer.cancer.gov/). Primary cancer site and histology were coded according to the third edition of the International Classification of Diseases for Oncology (ICD-O-3). We identified GIST patients using the "cs0204schema" variable. In the SEER dataset, codes 35 to 41 of the "cs0204schema" variable represent GIST tumors of the appendix, colon, esophagus, peritoneum, rectum, small intestine, and stomach, respectively. The exclusion criteria were as follows: diagnosis by autopsy or death certificate only, missing age, previous cancer history, distant metastasis, survival time of less than 1 month, missing data concerning tumor size and/or lymphadenectomy information. This study was approved by the Institutional Review Board of Sun Yat-sen University Cancer Center (ID: B2017-099-13).

\section{Exposure assessment}

We used the variable "EOD10_NE" to define lymphadenectomy. This variable describes the total number of regional lymph nodes removed and examined by the pathologist. This variable had 8 categories. Detailed information of each category can be found in Table S1. We defined the patients with codes $01-89,90$, and $96-98$ as the lymphadenectomy group; patients with codes 00 and 95 as the non-lymphadenectomy group; and patients with code 99 as the missing group. SEER defined tumor size information using two variables: "CSTUMSIZ" and "EOD10_SZ". We defined the tumor sizes of GIST patients diagnosed before 2003 using the variable "EOD10_SZ" and those after 2004 using "CSTUMSIZ". We coded continuous tumor size variables into 4 categories by defining the tumor size as less than 2, 2-5, 5-10, or $>10 \mathrm{~cm}$.

\section{Outcomes assessment}

Overall survival (OS) and cancer-specific survival (CSS) 


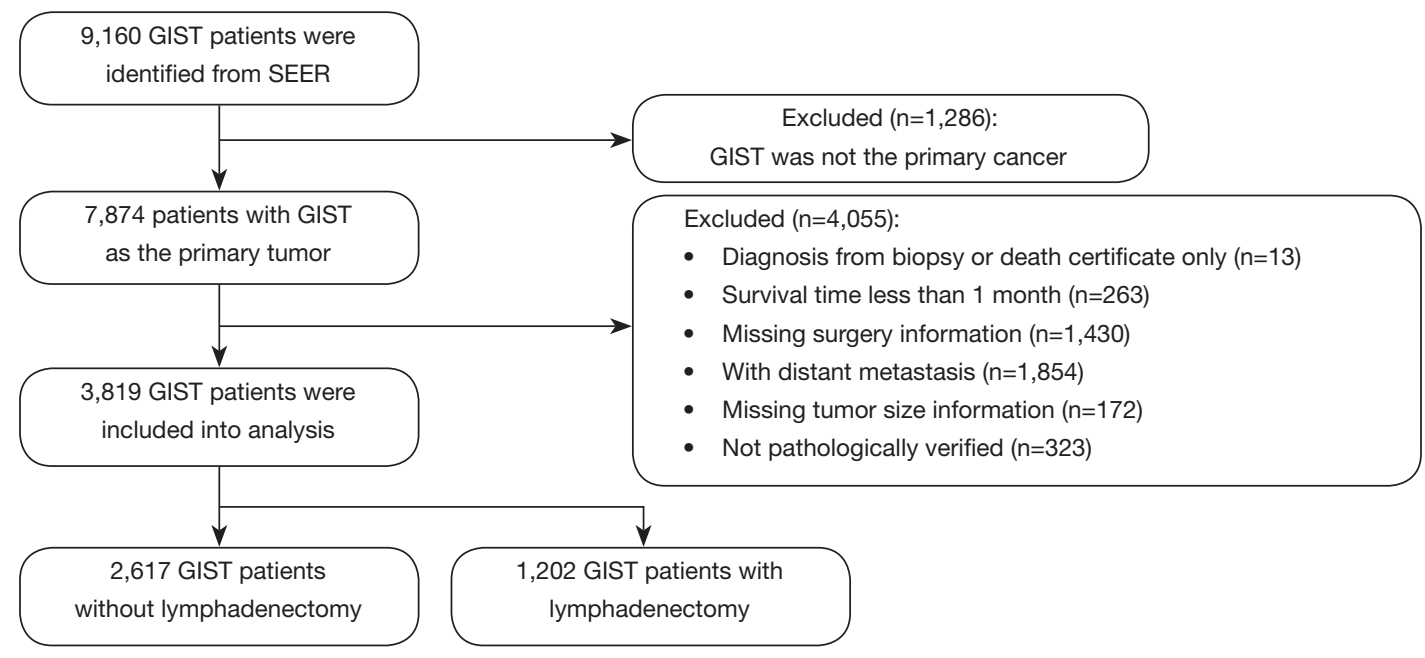

Figure 1 Flow chart of the analytic sample selection process.

were the outcomes of this study. We used "STAT_REC" and "VSRTSADX" variables to define the overall vital status and cancer-specific vital status, respectively. For the overall vital status, SEER recorded patients who died before the follow-up cut-off date as "death" and those after the cut-off date as "censored". The "VSRTSADX" variable designates the people who died as a result of their cancer as "cause-specific death" and the people who are still alive or who died from other cancers as "censored". We used the "srv_time_mon" variable to define survival time. The detailed calculation process of survival time can be found here: https://seer.cancer.gov/survivaltime/3-fields-survivaltime-active.pdf. Survival time was defined as the interval between the date of death or date of last contact and the date of GIST diagnosis. SEER records survival time in months.

\section{Assessment of potential confounders}

According to the literature review and our clinical experience, we included age at diagnosis (continuous), sex (male vs. female), race (non-Hispanic White, non-Hispanic Blacks, Asian Pacific Islanders, Hispanics, others), marital status (married vs. unmarried), and grade (I to IV) as potential confounding factors.

\section{Statistical analysis}

We used mean and standard deviations or medians and inter quartile ranges to describe continuous variables and proportions to describe categorical variables. We compared the distribution of social-demographic variables (e.g., age, sex, race, marital status) and clinical variables (e.g., grade) between the lymphadenectomy group and the nonlymphadenectomy group using $t$-tests or chi-square tests. For continuous data not normally distributed, we used Mann-Whitney $\mathrm{U}$ test instead of $t$-test. We tested the differences in OS and CSS between the lymphadenectomy group and the non-lymphadenectomy group using KaplanMeier methods with log-rank tests and Cox proportional regression models after adjusting for potential confounding factors. We also ran several further univariate and multivariate Cox regression analyses to examine whether the effects of lymphadenectomy on OS or CSS differed across various subgroups, including tumor size (less than 2, $2-5,5-10$, and $>10 \mathrm{~cm}$ ), tumor sites (colorectal, peritoneum, small intestine, stomach), and age (no more than 40 and above 40 years). All the tests were two-sided, 0.05 was set as the significance level. SAS 9.3 (SAS Institute, Cary, NC, USA) and R 3.2.1 were used for the data analysis.

\section{Results}

\section{Characteristics of the participants}

Figure 1 shows the participant flow chart selection process. Table 1 shows the social-demographic and clinical characteristics of the participants. A total of 3,819 GIST participants were included in the final analysis, 1,202 of whom accepted lymphadenectomy, and 2,617 of whom did not accept lymphadenectomy. The mean age of the 
Table 1 Social-demographic and clinical characteristics of the participants

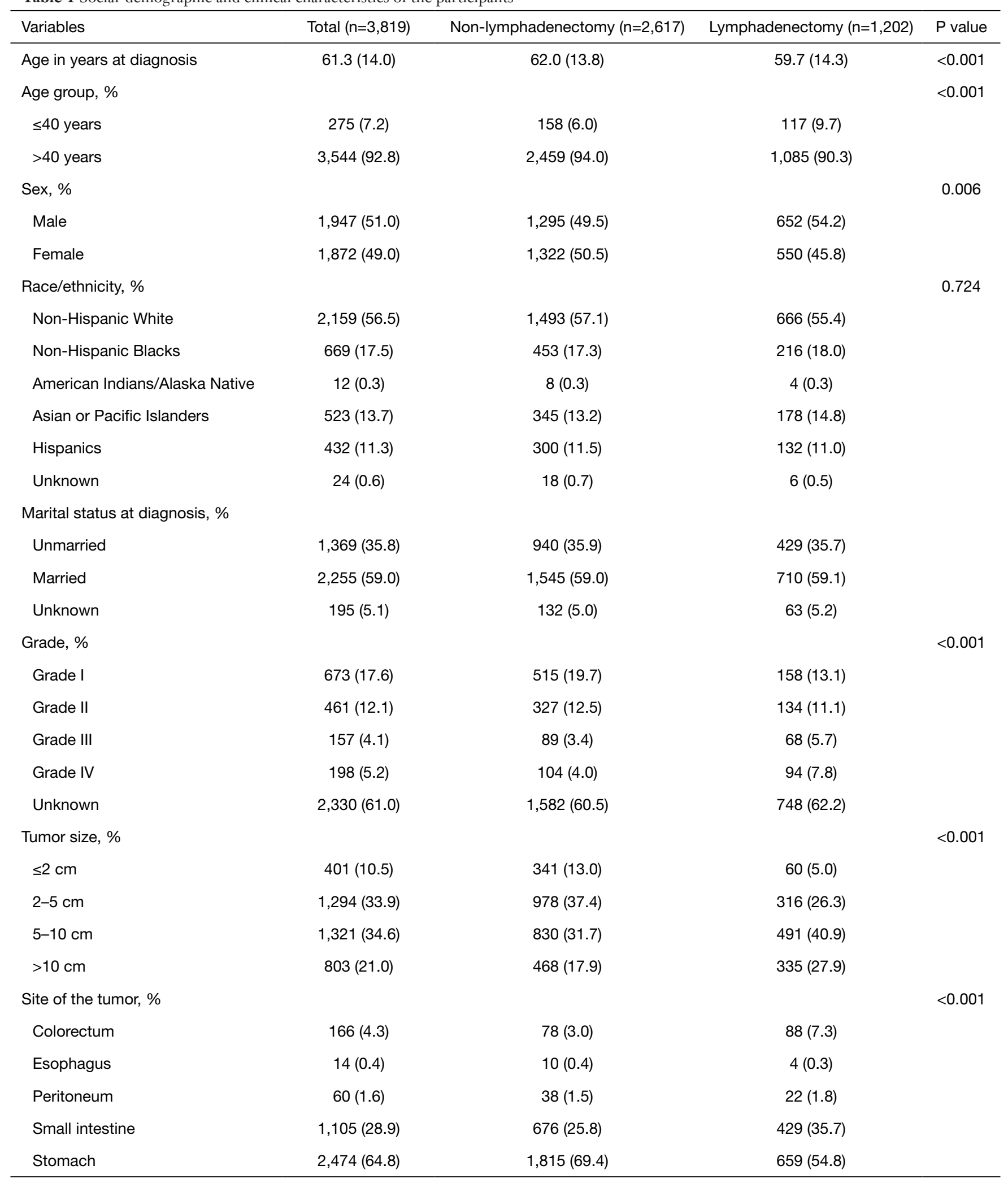




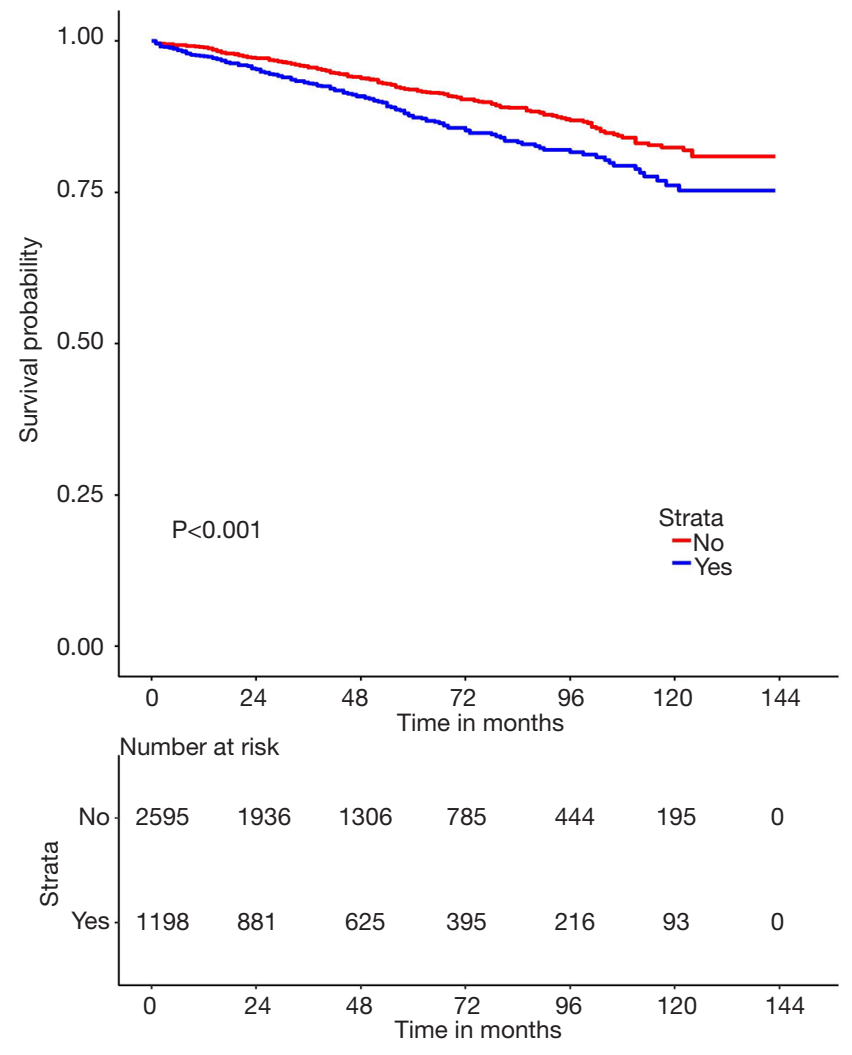

Figure 2 Association between lymphadenectomy and cancerspecific survival. No, no lymphadenectomy; Yes, lymphadenectomy.

participants was $61.3 \pm 14.0$ years. Most were more than 40 years old, over half $(51.0 \%)$ were male, $56.5 \%$ were nonHispanic White, and $59.0 \%$ were married. Most of the tumors occurred in the stomach $(64.8 \%)$ and were between $2-10 \mathrm{~cm}(68.5 \%)$. Compared with the lymphadenectomy group, the non-lymphadenectomy group were older (62.0 vs. 59.7 years), had a significantly higher proportion of females (50.5\% vs. $45.8 \%)$, and had smaller tumor sizes ( $<2 \mathrm{~cm}, 13.0 \%$ vs. $5.0 \%$ ). The lymphadenectomy group had more common sites in the stomach than the nonlymphadenectomy group (69.4\% vs. 54.8\%). Race/ethnicity and marital status between the groups were similar.

\section{Influence of lymphadenectomy on OS and CSS}

The median follow-up time was 49 months (range, 1-143 months), and 667 patients, accounting for $17.5 \%$ of the total, died. Median survival time was not reached for the total sample. The lymphadenectomy group had a significantly lower 5 -year OS (78.2\%, 95\% CI: $76.2-$
$80.1 \%$ vs. $81.8 \%$ 95\% CI: $79.8-83.8 \%)$ and CSS (85.6\%, 95\% CI: $83.7-87.6 \%$ vs. $90.4 \%$, 95\% CI: $88.4-92.4 \%)$ than the non-lymphadenectomy group (Figure 2). Our stratification analysis showed that lymphadenectomy was a poor prognostic factor for 5 -year OS and CSS among patients with a tumor size less than $10 \mathrm{~cm}$. For example, lymphadenectomy was associated with a $4.9 \%$ and $3.0 \%$ reduction in 5-year CSS among patients with a tumor size less than $2 \mathrm{~cm}$ and in patients with a tumor size between 5-10 cm, respectively. Among the patients with a tumor size greater than $10 \mathrm{~cm}$, we did not find any evidence lymphadenectomy improved their 5-year OS (70.2\%, 95\% CI: $65.2-75.3 \%$ vs. $71.3 \%, 95 \%$ CI: $65.3-77.2 \%)$ and CSS (77.6\%, 95\% CI: $72.9-82.4 \%$ vs. $78.1 \%$, 95\% CI: $72.5-83.8 \%)$. Our stratification analysis also showed that lymphadenectomy did not improve 5-year OS and CSS in GIST patients who were less than 40 years old. For GIST patients with stomach tumors, lymphadenectomy increased the risk of mortality $(\mathrm{P}<0.001)$. Figures 3 and 4 show the survival curves for the CSS of patients in the groups stratified by tumor sizes and tumor sites, respectively.

\section{Cox proportion analysis between lymphadenectomy and OS and CSS}

Table 2 shows the univariate and multivariate associations between lymphadenectomy and OS and CSS. We found that lymphadenectomy was associated with an increased risk of overall death (HR $=1.25,95 \%$ CI: $1.06-1.47)$ and cancer-specific death (HR $=1.32,95 \%$ CI: $1.07-1.64$ ) after adjusting for potential confounding factors. This phenomenon was especially evident among GIST patients with a tumor size less than $2 \mathrm{~cm}$ (CSS, HR $=6.37$, 95\% CI: 1.85-21.90). We also observed similar findings in patients with tumor sizes between $5-10 \mathrm{~cm}$ (overall death, adjusted $\mathrm{HR}=1.36,95 \%$ CI: 1.04-1.76; cancer-specific death, adjusted HR $=1.59,95 \%$ CI: $1.13-2.23)$. Strikingly, we found that lymphadenectomy did not improve OS (adjusted HR $=0.98,95 \%$ CI: $0.73-1.30$ ) or CSS (adjusted $\mathrm{HR}=0.85,95 \% \mathrm{CI}: 0.61-1.20)$ even in patients with a tumor size greater than $10 \mathrm{~cm}$. GIST patients more than 40 years old had poorer OS (HR $=1.28,95 \%$ CI: $1.08-1.51$ ) and CSS (HR $=1.36,95 \%$ CI: $1.09-1.70)$ than patients less than 40 years old after adjusting for potential confounding factors. Additionally, lymphadenectomy was associated with increased overall death (HR $=1.39,95 \%$ CI: $1.12-1.72$ ) and cancer-specific death (HR $=1.77,95 \%$ CI: $1.33-2.35$ ) among GIST patients with stomach tumors. 

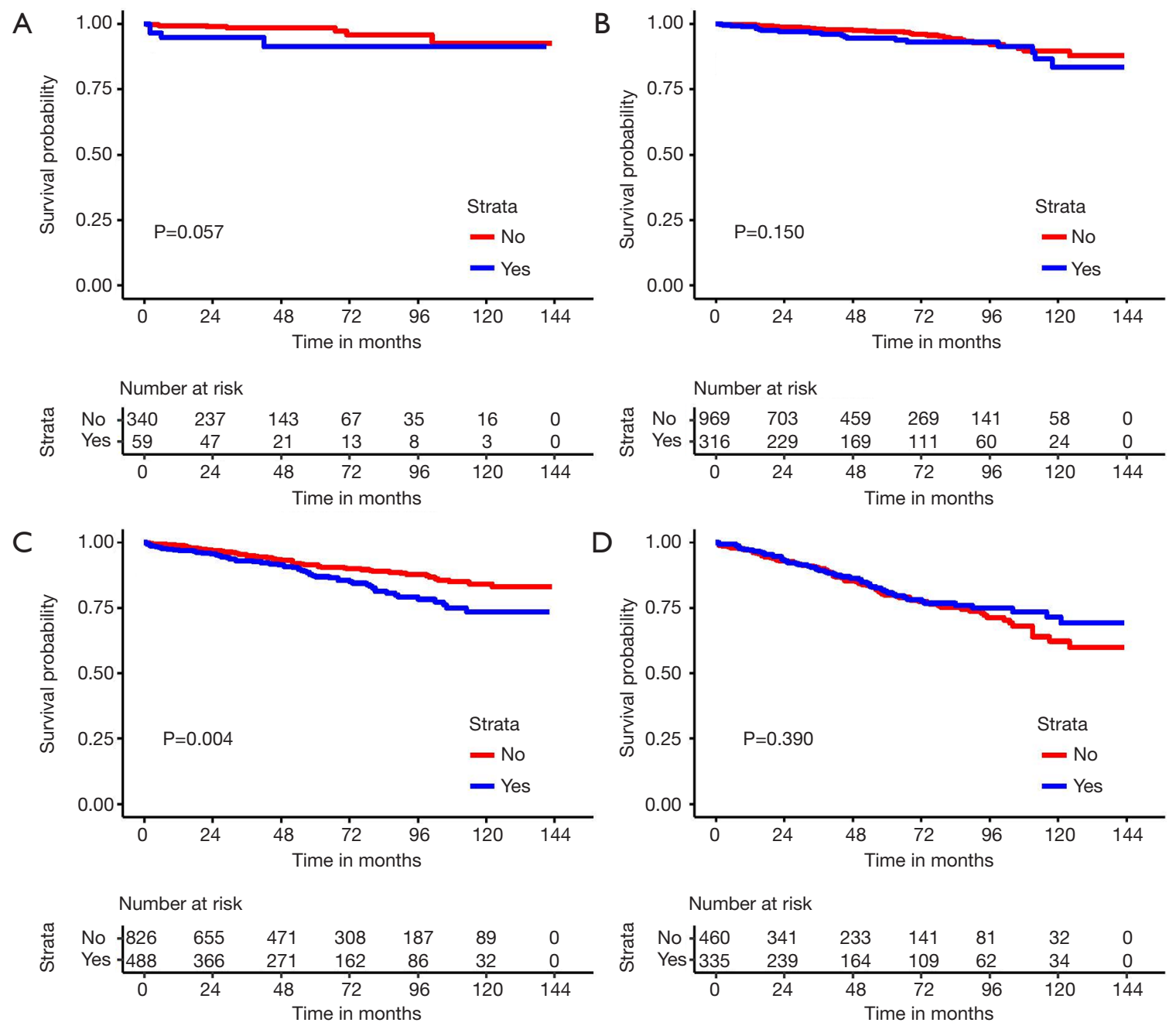

Figure 3 Association between lymphadenectomy and cancer-specific survival stratified by tumor sizes. (A) Tumor size $\leq 2 \mathrm{~cm}$; (B) tumor size between 2-5 cm; (C) tumor size between 5-10 cm; (D) tumor size >10 cm. No, no lymphadenectomy; Yes, lymphadenectomy.

\section{Discussion}

Our analysis shows that lymphadenectomy was associated with an increased risk of overall death and cancer-specific death in GIST patients. This was especially evident in patients with a primary tumor size of less than $2 \mathrm{~cm}$, who were more than 40 years old and had a stomach tumor. To our knowledge, this is the first study to assess the effect of lymphadenectomy on GIST patients' long-term survival.

The phenomenon that lymphadenectomy is associated with poor long-term survival in GIST patients may be explained by the following. First, nodal involvement is rare in GIST patients and unnecessary lymphadenectomy may destroy the immune micro-environment of the normal lymph nodes (10) causing a high risk of recurrence, which is one of the most important reasons for treatment failure in GIST patients after surgery within 2 years (11). Second, lymphadenectomy is associated with surgical trauma which increases postoperative morbidity and mortality. Contrary to our hypothesis, we did not find that lymphadenectomy improves GIST patients' long-term survival even among patients with a tumor size greater than $10 \mathrm{~cm}$, which is perceived as an important indicator for lymph nodes metastasis. This seemingly paradoxical phenomenon could also be explained by the low metastatic rate of lymph nodes for this disease. Naguib et al. performed lymph node dissection on 5 out of 19 patients with gastric GIST and found that none of the dissected lymph nodes were cancer 
A
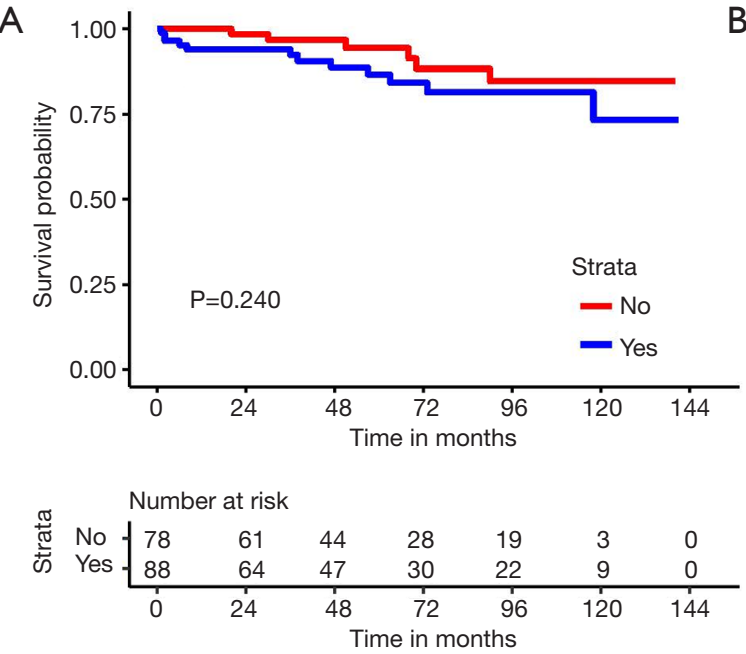

C
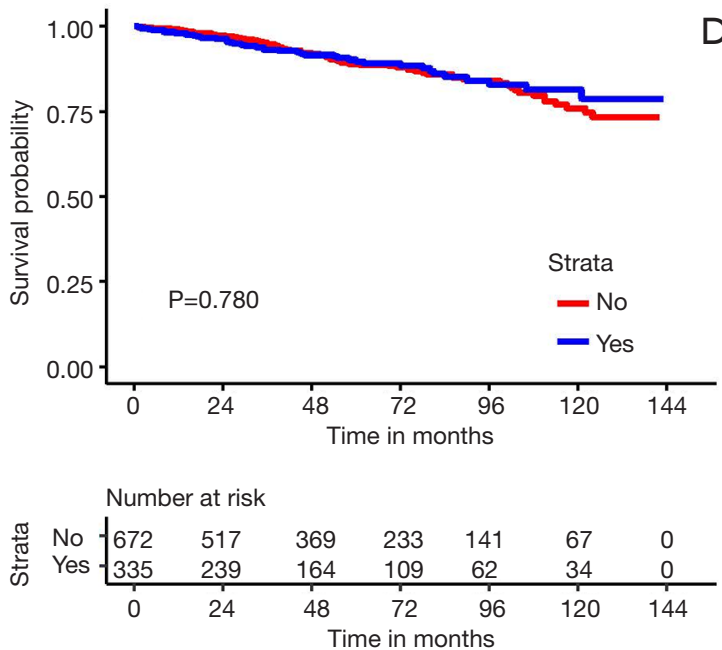

$B$
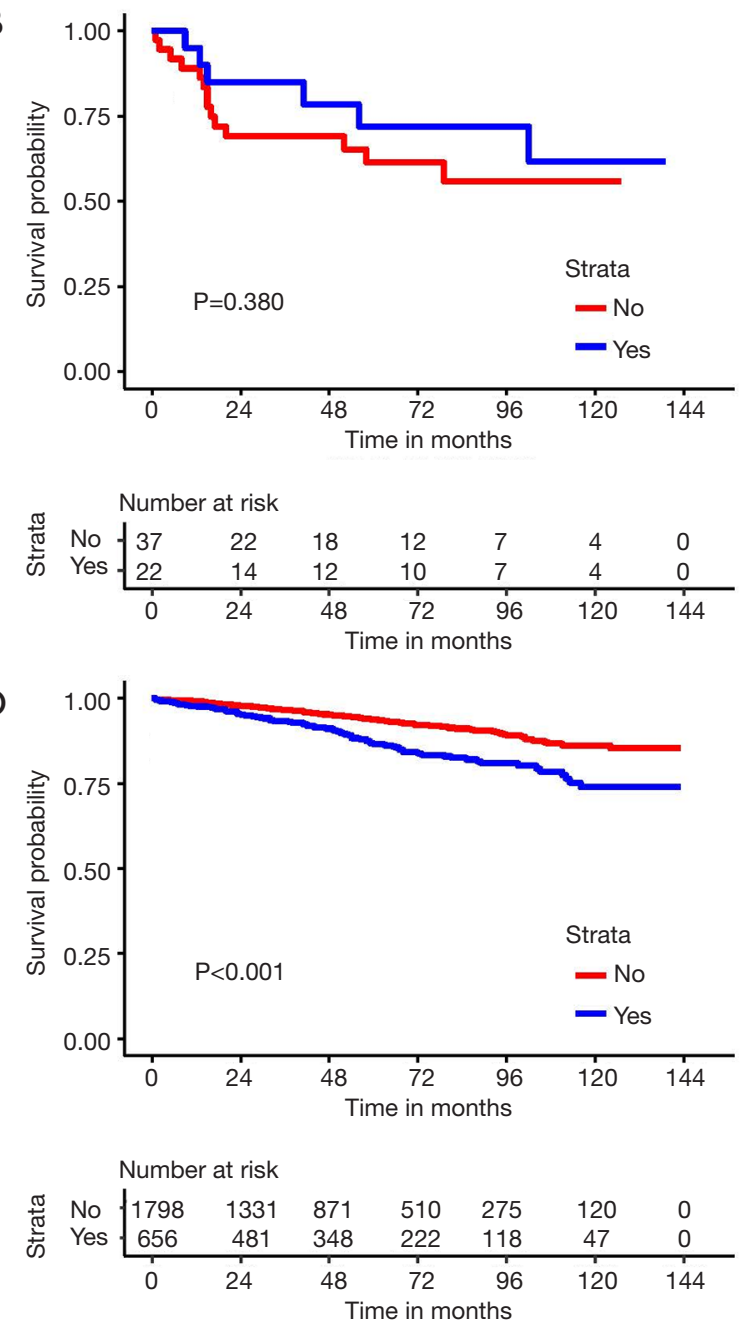

Figure 4 Association between lymphadenectomy and cancer-specific survival stratified by tumor sites. (A) Colorectum; (B) peritoneum; (C) small intestine; (D) stomach. No, no lymphadenectomy; Yes, lymphadenectomy.

cell positive (12). Similarly, our supplemental analysis showed that over $90 \%$ of the dissected lymph nodes were cancer cell negative. There were no significant differences in the rate of nodal involvement between GIST patients with a tumor size greater than $10 \mathrm{~cm}$ or those with a tumor size less than $2 \mathrm{~cm}$. Therefore, irrespective of GIST tumor size, lymphadenectomy appears to be.an unnecessary treatment for most GIST patients.

The rate of nodal involvement differed significantly across age groups of GIST patients. Agaimy and Wunsch reported that the rate of regional node metastasis for gastric patients who were less than 40 years old was $25 \%$, while for the whole population the metastatic rate was only $1 \%$ (5). In this study, we found, contrary to our hypothesis, that lymphadenectomy does not improve GIST patients' longterm survival among those less than 40 years old. This indicates that lymphadenectomy may be an unnecessary treatment even among patients with a relatively high risk of lymph node metastasis. Our finding is similar to a previous study which revealed that metastasis had no impact on overall and disease-free survival in GIST patients (13).

The lymphatic drainage system of the GI tract is complex (14). The number of lymph nodes and the possibilities of nodal involvement could differ greatly across different organs. Evidence has suggested that the metastatic rate for small intestinal GIST is significantly higher than other types of GIST tumors. In this study, we found that lymphadenectomy does not improve long-term survival 
Table 2 Association between lymphadenectomy and OS and CSS among non-metastatic GIST patients in the SEER dataset

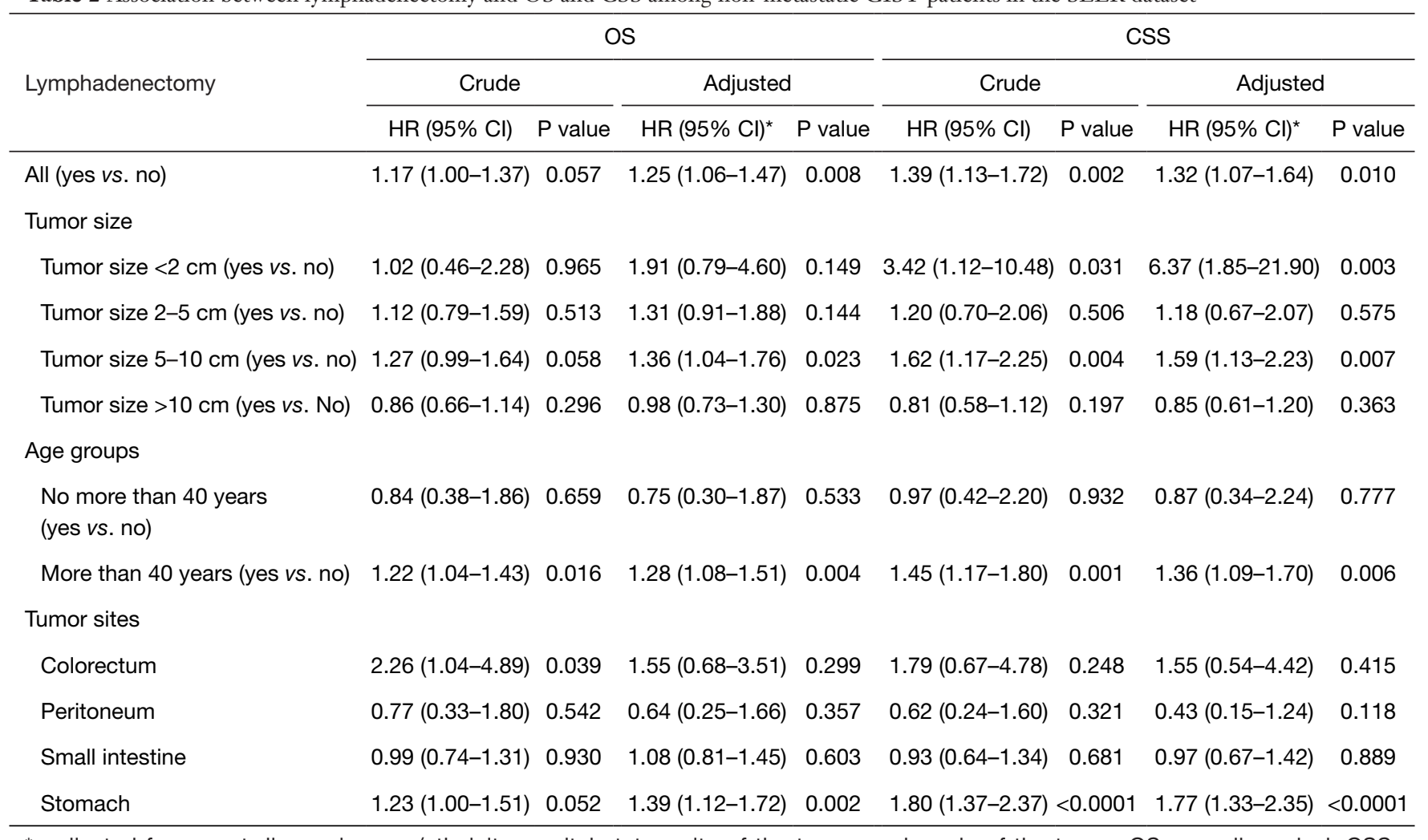

*, adjusted for age at diagnosis, race/ethnicity, marital status, site of the tumor, and grade of the tumor. OS, overall survival; CSS, cancer-specific survival; GIST, gastrointestinal stromal tumor; SEER, Surveillance, Epidemiology, and End Results.

among GIST patients with tumors in the colorectum, esophagus, peritoneum, and small intestine, and that those with stomach tumors had an increased risk of overall and CSS death. Although we are unsure as to why, we suspect this is because most epithelioid GISTs were benign, with a mitotic count of less than 5/50 HPFs (15). Another possible explanation is the small sample sizes of patients for other types of GIST tumors. Ultimately, the findings of this study do not support lymphadenectomy according to those tumor sites.

Our study has several limitations. First, we did not adjust for the use of imatinib and mitotic rate, as SEER does not collect imatinib information, and a large percent of data concerning the mitotic rate is missing. Second, all participants were from the USA, so the findings are not generalizable to other populations. Therefore, caution and further analyses are needed before conclusions can be made to other populations.

\section{Conclusions}

In summary, in contrast to what was previously assumed, lymphadenectomy was associated with an increased and not a decreased, risk of mortality in GIST patients. In this study, primary tumor size and age were seemingly not indictors for lymphadenectomy. Further research, however, is needed before claims of possible lymphadenectomyrelated over-treatment can be substantiated.

\section{Acknowledgments}

None.

\section{Footnote}

Conflicts of Interest: The authors have no conflicts of interest to declare. 
Ethical Statement: The authors are accountable for all aspects of the work in ensuring that questions related to the accuracy or integrity of any part of the work are appropriately investigated and resolved. This study was approved by the Institutional Review Board of Sun Yat-sen University Cancer Center (ID: B2017-099-13).

\section{References}

1. Rubin BP, Heinrich MC, Corless CL. Gastrointestinal stromal tumour. Lancet 2007;369:1731-41.

2. Burkill GJ, Badran M, Al-Muderis O, et al. Malignant gastrointestinal stromal tumor: distribution, imaging features, and pattern of metastatic spread. Radiology 2003;226:527-32.

3. Gaitanidis A, El LM, Alevizakos M, et al. Predictors of lymph node metastasis in patients with gastrointestinal stromal tumors (GISTs). Langenbecks Arch Surg 2018;403:599-606.

4. Roggin KK, Posner MC. Modern treatment of gastric gastrointestinal stromal tumors. World J Gastroenterol 2012;18:6720-8.

5. Agaimy A, Wunsch PH. Lymph node metastasis in gastrointestinal stromal tumours (GIST) occurs preferentially in young patients $<$ or $=40$ years: an overview based on our case material and the literature. Langenbecks Arch Surg 2009;394:375-81.

6. Gastrointestinal stromal tumours: ESMO Clinical Practice Guidelines for diagnosis, treatment and follow-up. Ann Oncol 2014;25 Suppl 3:i21-6.

7. Hartgrink HH, van de Velde CJ, Putter H, et al. Extended lymph node dissection for gastric cancer: who may benefit?
Final results of the randomized Dutch gastric cancer group trial. J Clin Oncol 2004;22:2069-77.

8. Chen M, Palleschi S, Khoynezhad A, et al. Role of primary breast cancer characteristics in predicting positive sentinel lymph node biopsy results: a multivariate analysis. Arch Surg 2002;137:606-9, 609-10.

9. Park HS, Lloyd S, Decker RH, et al. Overview of the Surveillance, Epidemiology, and End Results database: evolution, data variables, and quality assurance. Curr Probl Cancer 2012;36:183-90.

10. Vuletic A, Jovanic I, Jurisic V, et al. Decreased Interferon gamma Production in CD3+ and CD3CD56+ Lymphocyte Subsets in Metastatic Regional Lymph Nodes of Melanoma Patients. Pathol Oncol Res 2015;21:1109-14.

11. Dematteo RP, Ballman KV, Antonescu CR, et al. Adjuvant imatinib mesylate after resection of localised, primary gastrointestinal stromal tumour: a randomised, doubleblind, placebo-controlled trial. Lancet 2009;373:1097-104.

12. Naguib SF, Zaghloul AS, El MH. Gastrointestinal stromal tumors (GIST) of the stomach: retrospective experience with surgical resection at the National Cancer Institute. J Egypt Natl Canc Inst 2008;20:80-9.

13. Valadao M, de Mello EL, Lourenco L, et al. What is the prognostic significance of metastatic lymph nodes in GIST? Hepatogastroenterology 2008;55:471-4.

14. Alexander JS, Ganta VC, Jordan PA, et al. Gastrointestinal lymphatics in health and disease. Pathophysiology 2010;17:315-35.

15. Miettinen M, El-Rifai W, H L Sobin L, et al. Evaluation of malignancy and prognosis of gastrointestinal stromal tumors: a review. Hum Pathol 2002;33:478-83.
Cite this article as: Li C, Su D, Xie C, Chen Q, Zhou J, $\mathrm{Wu} \mathrm{X}$. Lymphadenectomy is associated with poor survival in patients with gastrointestinal stromal tumors. Ann Transl Med 2019;7(20):558. doi: 10.21037/atm.2019.09.60 
Supplementary

Table S1 The categories of "EOD10_NE" in the Surveillance, Epidemiology, and End Results database

\begin{tabular}{|c|c|c|}
\hline Code & Description & Lymphadenectomy \\
\hline 01-89 & Exact number of nodes examined & Yes \\
\hline 90 & 90 or more nodes were examined & Yes \\
\hline 95 & No regional nodes were removed, but aspiration of regional nodes was performed & No \\
\hline 97 & $\begin{array}{l}\text { Regional lymph node removal was documented as a dissection, and the number of nodes is unknown/not } \\
\text { stated }\end{array}$ & Yes \\
\hline 98 & $\begin{array}{l}\text { Regional lymph nodes were surgically removed, but the number of lymph nodes is unknown/not stated and } \\
\text { not documented as a sampling or dissection; nodes were examined, but the number is unknown }\end{array}$ & Yes \\
\hline
\end{tabular}

